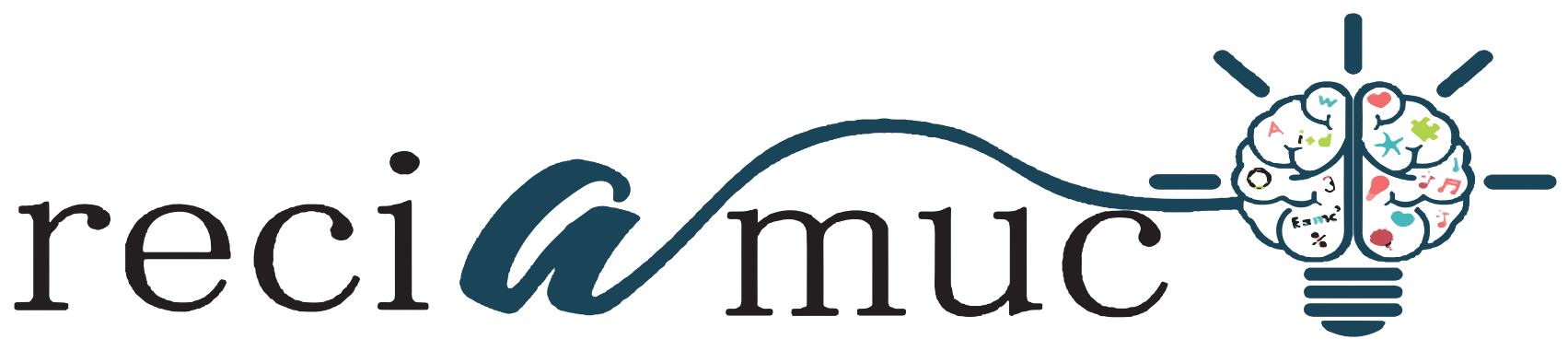

DOI: $10.26820 /$ reciamuc/5.(3).agosto.2021.36-43

URL: https://reciamuc.com/index.php/RECIAMUC/article/view/683

EDITORIAL: Saberes del Conocimiento

REVISTA: RECIAMUC

ISSN: 2588-0748

TIPO DE INVESTIGACIÓN: Artículo de Revisión

CóDIGO UNESCO: 32 Ciencias Médicas

PAGINAS: $36-43$

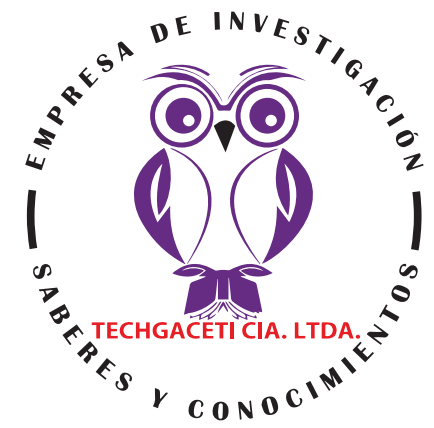

\title{
Lesiones dermatológicas en pediatría por infección SARS-CoV-2
}

Dermatological lesions in pediatrics due to SARS-CoV-2 infection

Lesões dermatológicas em pediatria devido à infecção por SARS-CoV-2

\section{Viviana del Rocío Moreira Quiroz'; Cinthia Alexandra Romero Tejada2; María Dolores Zambrano Zambrano3; María Isabel Zambrano Zambrano ${ }^{4}$}

RECIBIDO: 10/06/2021 ACEPTADO: 12/07/2021 PUBLICADO: 31/08/2021

1. Doctora en Medicina y Cirugía; Médico General en funciones Hospitalarias; Servicio de Pediatría en Hospital Verdi Cevallos Balda de Portoviejo; Ecuador; vivirociomoreira@gmail.com; iD https://orcid.org/0000-00016071-8864

2. Médico; Médico General en funciones Hospitalaria; Servicio Pediatría-Neonatología en Hospital Verdi Cevallos Balda de Portoviejo; Ecuador; cinthya_tejada@hotmail.com; (iD) https://orcid.org/0000-0002-9119-4909

3. Médico Cirujano; Médico General en funciones Hospitalaria; Servicio Pediatría; Ecuador; maloly_1984@hotmail.com; iD https://orcid.org/0000-0001-8504-1327

4. Médico Cirujano; Médico General en funciones Hospitalaria; Servicio Pediatría - Neonatología en Hospital Verdi Cevallos Balda de Portoviejo; Ecuador; maisabelzamb@hotmail.com; iD https://orcid.org/0000-0003-19016806

\section{CORRESPONDENCIA}

Viviana del Rocío Moreira Quiroz vivirociomoreira@gmail.com

Portoviejo; Ecuador 


\section{RESUMEN}

Desde diciembre de 2019 el mundo se ha visto afectado por una pandemia causada por el virus SARS-CoV-2, causante de la enfermedad COVID-19, conocida por su gran capacidad infecciosa y rápida transmisión. Este virus se transmite por contacto de persona a persona y a través de secreciones de personas infectadas, principalmente gotitas respiratorias. Desde el inicio de la pandemia se conoce que los niños, niñas y adolescentes tienen un menor riesgo de enfermarse y de morir por COVID-19 que otros grupos etarios. Las manifestaciones clínicas de la COVID-19 son múltiples y variadas, y son muy parecidas a las del resfriado común. Últimamente se han descrito lesiones cutáneas inespecíficas, más frecuentemente en niños y adolescentes, a veces como único síntoma clínico o en el contexto del cuadro típico de la infección. Entre las manifestaciones cutáneas registradas en pacientes con COVID-19 incluyen, exantemas urticarianos, morbiliformes, vesiculares y petequiales, así como manifestaciones vasoespásticas como livedo reticularis, lesiones isquémicas acrales y lesiones cutáneas en los dedos de los pies y manos idénticas a los sabañones. El tratamiento es sintomático, pudiendo manejarse con corticoides tópicos, antihistamínicos en el caso de prurito moderado, y antibióticos tópicos para evitar sobreinfecciones en las lesiones acroisquémicas. El enfoque general del presente trabajo es dar a conocer las principales lesiones dermatológicas ocasionadas por la infección SARS-CoV-2 en pacientes pediátricos, sus características, síntomas y tratamientos. Los resultados se obtuvieron mediante el desarrollo de una investigación de tipo bibliográfica, delimitada a una metodología de revisión, que permite concluir que aun y cuando este tipo de lesiones no representan una amenaza grave para los pacientes, se recomienda conocer la sintomatología de las mismas para así aplicar las medidas preventivas y de tratamiento que eviten posibles complicaciones.

Palabras clave: Lesiones dermatológicas, manifestaciones cutáneas, infección, paciente pediátrico, SARS-CoV-2, COVID-19.

\section{ABSTRACT}

Since December 2019, the world has been affected by a pandemic caused by the SARS-CoV-2 virus, which causes the COVID-19 disease, known for its high infectious capacity and rapid transmission. This virus is transmitted by person-to-person contact and through secretions of infected people, mainly respiratory droplets. Since the beginning of the pandemic, it has been known that children and adolescents have a lower risk of becoming ill and dying from COVID-19 than other age groups. The clinical manifestations of COVID-19 are multiple and varied, and are very similar to those of the common cold. Lately, nonspecific skin lesions have been described, more frequently in children and adolescents, sometimes as the only clinical symptom or in the context of the typical picture of the infection. Among the skin manifestations recorded in patients with COVID-19 include urticarial, morbilliform, vesicular and petechial rashes, as well as vasospastic manifestations such as livedo reticularis, acral ischemic lesions, and skin lesions on the fingers and toes identical to chilblains. Treatment is symptomatic and can be managed with topical corticosteroids, antihistamines in the case of moderate itching, and topical antibiotics to avoid superinfections in acroischemic lesions. The general approach of this work is to present the main dermatological lesions caused by SARS-CoV-2 infection in pediatric patients, their characteristics, symptoms and treatments. The results were obtained through the development of a bibliographic type research, limited to a review methodology, which allows to conclude that even though this type of injury does not represent a serious threat to patients, it is recommended to know the symptoms of the same to thus apply preventive and treatment measures that avoid possible complications.

Keywords: Dermatological lesions, skin manifestations, infection, pediatric patient, SARS-CoV-2, COVID-19.

\section{RESUMO}

Desde dezembro de 2019, o mundo é afetado por uma pandemia causada pelo vírus SARS-CoV-2, causador da doença COVID-19, conhecida por sua alta capacidade infecciosa e rápida transmissão. Este vírus é transmitido por contato pessoa a pessoa e por secreções de pessoas infectadas, principalmente gotículas respiratórias. Desde o início da pandemia, sabe-se que crianças e adolescentes têm menor risco de adoecer e morrer de COVID-19 do que outras faixas etárias. As manifestações clínicas da COVID-19 são múltiplas e variadas e muito semelhantes às do resfriado comum. Ultimamente, lesões cutâneas inespecíficas têm sido descritas, mais freqüentemente em crianças e adolescentes, às vezes como o único sintoma clínico ou no contexto do quadro típico da infecção. Entre as manifestações cutâneas registradas em pacientes com COVID-19 incluem erupções urticariformes, morbiliformes, vesiculares e petéquias, bem como manifestações vasoespásticas, como livedo reticular, lesões isquêmicas acrais e lesões cutâneas nos dedos das mãos e dos pés idênticas às frieiras. O tratamento é sintomático e pode ser administrado com corticosteroides tópicos, anti-histamínicos no caso de coceira moderada e antibióticos tópicos para evitar superinfecções em lesões acroquímicas. A abordagem geral deste trabalho é apresentar as principais lesões dermatológicas causadas pela infecção por SARS-CoV-2 em pacientes pediátricos, suas características, sintomas e tratamentos. Os resultados foram obtidos através do desenvolvimento de uma pesquisa do tipo bibliográfico, limitada a uma metodologia de revisão, que permite concluir que mesmo que este tipo de lesão não represente uma ameaça grave aos pacientes, recomenda-se conhecer a sintomatologia da mesma. assim, aplicar medidas preventivas e de tratamento que evitem possíveis complicações.

Palavras-chave: Lesões dermatológicas, manifestações cutâneas, infecção, paciente pediátrico, SARS-CoV-2, COVID-19. 
NO, M. I.

\section{Introducción}

Desde diciembre de 2019 el mundo se ha visto afectado por una pandemia causada por el virus SARS-CoV-2, causante de la enfermedad COVID-19, conocida por su gran capacidad infecciosa y rápida transmisión. Este virus "se transmite por contacto de persona a persona y a través de secreciones de personas infectadas, principalmente gotitas respiratorias" (Ruiz \& Jimenez, 2020).

La Organización Panamericana de la Salud (OPS) (2021) refiere que:

Desde la confirmación de los primeros casos de COVID-19 hasta el 17 de mayo de 2021, fueron notificados 162.773 .940 casos acumulados confirmados de COVID-19, incluidas 3.375.573 defunciones en todo el mundo, lo que representa un total de 26.658 .506 casos confirmados adicionales de COVID-19, incluidas 438.657 defunciones, desde la última actualización epidemiológica publicada por la OPS/OMS el 14 de abril de 2021.

Aun y cuando la sintomatología tanto en los adultos, como en los niños se asemeja a la de la gripe o resfriado común, el virus se caracteriza por ser menos graves en los pacientes pediátricos que en los adultos. "Los síntomas más comunes de COVID-19 en pediatría incluyen fiebre, tos, rinorrea, vómito, diarrea y mialgias, además hay un menor porcentaje de dificultad respiratoria si se compara con los adultos" Benavides et al. (2020).

Adicional a los síntomas respiratorios característicos de la enfermedad, se han registrado manifestaciones cutáneas, con similitud clínica entre niños y adultos. Entre las que Granados et al. (2020) menciona las siguientes:
1. Erupción máculo-papular.
2. Urticaria.

3. Lesiones acrales tipo perniciosas.

4. Erupción vesicular.

5. Livedo reticularis.

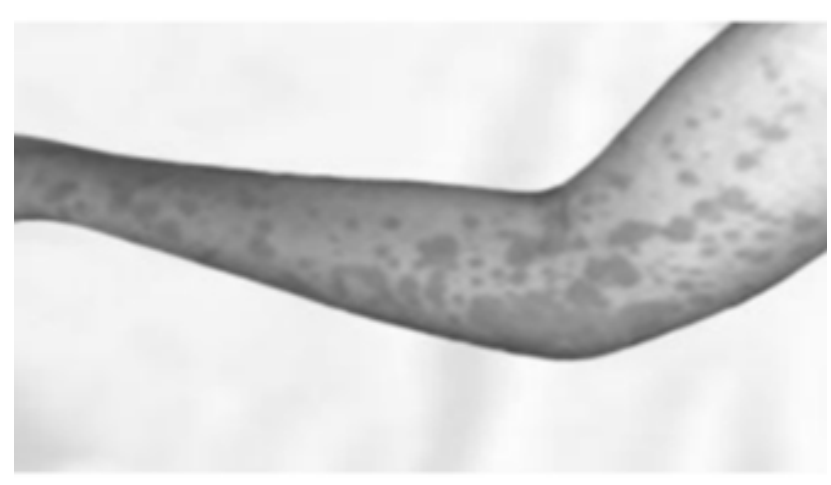

Figura 1. Urticaria.

Nota. Recuperado de: Lesiones habonosas en la cara anterolateral del brazo. Granados et al. (2020). Revista Cubana de Pediatría 92.

Recuperado de: http://www.revpediatria. sld.cu/index.php/ped/article/view/1171/556

Dada la situación actual, es de especial importancia conocer esta sintomatología como parte del cuadro clínico de la enfermedad, para así sospechar y por ende diagnosticar correctamente, tomando todas las medidas necesarias para una mejor atención y menor propagación de la enfermedad.

El objetivo de este trabajo investigativo, desarrollado bajo un metodología documental, se centra en realizar una revisión a la literatura científica disponible, que principalmente aborde a las lesiones dermatológicas en pacientes pediátricos causadas por infección con SARS-CoV-2, sus principales características y síntomas, así como los diversos tratamientos a aplicar, todo ello con la finalidad de proporcionar un material actualizado que defina dichos aspectos y que sea de utilidad no solo para el personal de salud, sino también para el público en ge- 
neral, por lo que para ello se estructura de la siguiente manera: definiciones, medios de transmisión, sintomatología, características y tratamiento.

\section{Materiales y métodos}

El presente trabajo de investigación, enmarcado en una metodología de revisión, está orientado a la construcción de un material bibliográfico actualizado, enfocado en dar a conocer los criterios vigentes de las lesiones dermatológicas en pediatría por infección SARS-CoV-2.

Entre las bases de datos consultadas destacan: Organización Panamericana de la Salud (PAHO), Scielo, medigraphic, mayo clinic, entre otras. Como términos de búsqueda se utilizaron las expresiones "SARS-CoV-2 en pacientes pediátricos", "Lesiones Dermatológicas en pediatría por SARS-CoV-2", "Manifestaciones Cutáneas en pediatría por SARS-CoV-2", y se aplicaron criterios de selección tales como: idioma español; publicación entre 2020 y 2021 (ambos inclusive); acceso completo y abierto; en el área de salud y medicina; estudios referidos a humanos; tipo de bibliografía, manuales médicos, guías clínicas, ensayos clínicos, estudios o reportes de casos, boletines y/o folletos de instituciones oficiales o privadas de excelente trayectoria en el área de la salud, medicina o científico académica, y demás, monografías y otros documentos que, a criterio propio, mostraran información de interés en base a la observación de la evidencia científica referida en sus contenidos. Este proceso arrojó resultados que en promedio oscilaron entre 8 y 72 enlaces a fuentes de información bibliográficas.

Es a partir de entonces que se procedió con la lectura crítica y análisis interpretativo de un pilar de información recopilado a lo largo de la investigación, que también fue adoptada como evidencia. Resultando todo este proceso en la selección de los elementos más sustanciales y significativos de las diferentes fuentes bibliográficas que fundamentan el razonamiento aquí expuesto.

\section{Resultados}

Un nuevo tipo de coronavirus llamado SARS-CoV-2 causante de la enfermedad COVID-19, la cual se manifiesta como una infección del tracto respiratorio, ha sido el motivo de un estado de alarma a nivel mundial.

"EI SARS-CoV-2 es un virus ARN perteneciente a la familia Coronaviridae. No se conoce con certeza la fuente del virus, pero se relaciona con animales salvajes como el murciélago y reservorios intermediarios como el pangolín, serpientes y visones". Solano et al. (2020).

Este virus ha sido catalogado como pandemia y ha generado a nivel mundial no solo una crisis de salud, sino una amenaza social y económica, creando situaciones extremadamente desafiantes, particularmente para los más vulnerables.

\section{SARS-COV-2 en Pediatría}

Desde el inicio de la pandemia se conoce que los niños, niñas y adolescentes tienen un menor riesgo de enfermarse y de morir por COVID-19 que otros grupos etarios. "Los niños presentan menos infecciones y menos gravedad que los adultos. La forma de presentación más frecuente en la edad pediátrica es la enfermedad leve; sin embargo, lactantes y preescolares han presentado de forma ocasional, cuadros graves" (Oliva, 2021)

\section{Transmisión}

Con los primeros reportes de infección por SARS-CoV-2 fue posible identificar que la infección es transmitida de una persona a otra a través del contacto cercano y tanto la población inmunocompetente como inmunocomprometida.

Las principales rutas de transmisión tanto en adultos como en niños fueron descritas por Vargas et al. (2020): 
NO, M. I.

1. Transmisión por gotas: ocurre cuando una persona infectada tose o estornuda y las gotas liberadas son ingeridas o inhaladas por personas cercanas.

2. Transmisión por contacto: cuando un sujeto tiene contacto con superficies $u$ objetos contaminados con el virus y posteriormente se toca la boca, la nariz o los ojos.

3. Transmisión por aerosoles: sucede cuando las gotas respiratorias se mezclan en el aire del ambiente de un lugar relativamente cerrado, formando aerosoles que se inhalan en altas dosis causando infección.

4. Transmisión de una persona a otra entre miembros de la familia: incluidos familiares y amigos que han estado en contacto íntimo con pacientes o portadores de incubación.

\section{Sintomatología}

Si bien los niños y los adultos experimentan síntomas similares de la COVID-19, los síntomas de los niños tienden a ser leves y parecidos a los del resfriado común. Las manifestaciones clínicas en niños reportadas hasta el momento incluyen las referidas por Montaño \& Miranda (2021):

1. Fiebre.

2. Tos habitualmente seca.

3. Fatiga.

4. Rinorrea.

5. Congestión Nasal.

6. Cefalea/Irritabilidad.

7. Cianosis.

8. Hiporexia/disminución de la ingesta.

9. Hipo actividad.

10. Rechazo al alimento.

11. Diarrea y otros síntomas gastrointes- tinales (nausea y vomito).

12. Exantema maculopapular generalizado.

De acuerdo con lo observado en diversos estudios realizados, la mayoría de los niños que se infectan con el virus de la COVID-19 tienen apenas una enfermedad leve, sin embargo, algunos pueden evolucionar y requerir cuidados intensivos, tal es el caso de los niños que desarrollan el síndrome inflamatorio multisistémico pediátrico (MIS-C), entre cuyos síntomas que advierten que se trata de una complicación grave destacan los referidos por Mayo Clinic (2021):

- Dolor intenso de estómago

- Dificultad para respirar

- Piel, labios o lecho de las uñas de coIor pálido, grisáceo o azulado, según el tono normal de la piel

- Confusión repentina

- Incapacidad para despertarse o permanecer despierto

\section{Lesiones Dermatológicas}

Las manifestaciones clínicas de la COVID-19 son múltiples y variadas. Últimamente se han descrito lesiones cutáneas inespecíficas, más frecuentemente en niños y adolescentes, a veces como único síntoma clínico o en el contexto del cuadro típico de la infección.

Entre los hallazgos cutáneos observados en pacientes pediatricos, se encuentran los descritos por Reyes (2021):

- Lesiones pseudosabañón: máculas, placas y nódulos rojo-violáceos en áreas acrales (pies y manos).

- Erupciones vesiculares tipo vesícula like: por lo general son pequeñas vesículas que aparecen en el tronco y las extremidades. No afectan a las mucosas.

- Lesiones tipo urticarias: son pruriginosas y mayormente se distribuyen en el 
tronco o son dispersas.

- Erupciones maculopapulares: se describen lesiones de distribución perifolicular y descamación, lesiones pitiriasis rosada-like, lesiones purpúricas puntiforme o en grandes áreas, pápulas pseudovesiculares en las extremidades y lesiones de eritema multiforme.

- Livedo o necrosis: En el tronco o las áreas acrales.

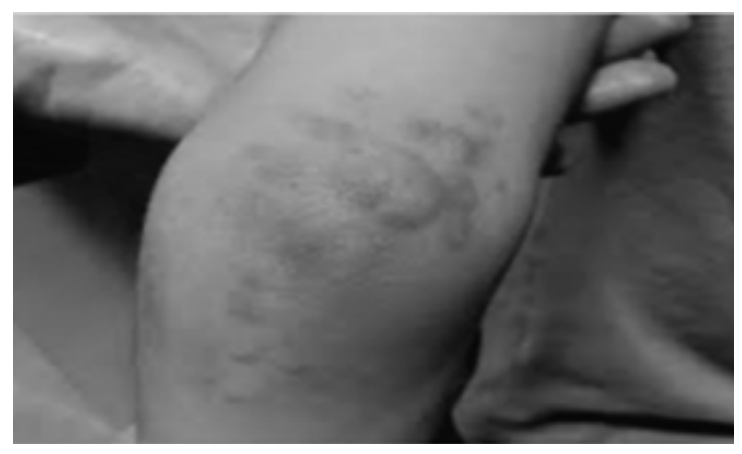

Figura 2. Erupciones Maculopapulares.

Nota. Recuperado de: Máculo-pápulas de aspecto discoide sugerente de eritema multiforme. Reyes. (2021). Acta Pediátrica México 42(1). Pág. 48-52

Recuperado de: https://ojs.actapediatrica.org.mx/index.php/APM/article/download/2215/1234

Otras manifestaciones cutáneas registradas en pacientes con COVID-19 incluyen "exantemas urticarianos, morbiliformes, vesiculares y petequiales, así como manifestaciones vasoespásticas como livedo reticularis, lesiones isquémicas acrales y lesiones cutáneas en los dedos de los pies y manos idénticas a los sabañones" Andina et al. (2020).

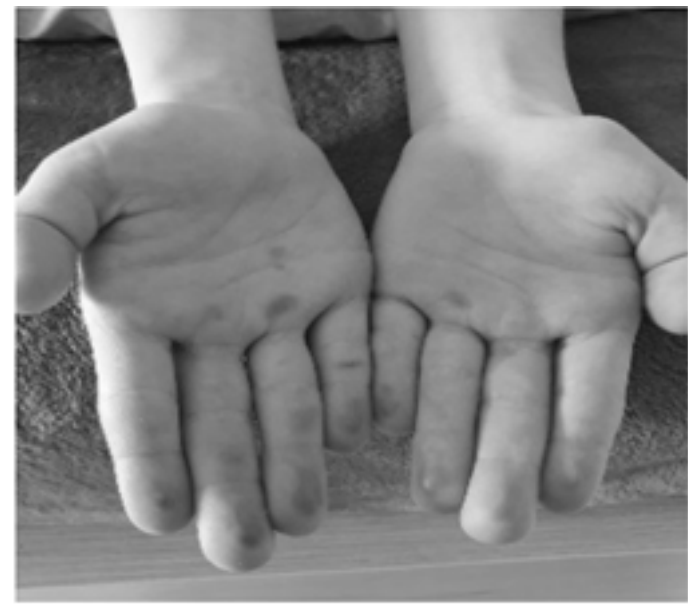

Figura 3. Lesiones isquémicas acrales.

Nota. Recuperado de: Acroisquemia por COVID-19. Ordoñez \& Domínguez (2020). Pediatría Atención Primaria 2(87). Pág. 295-303

Recuperado de: https://pap.es/articulo/13107/acroisquemia-aguda-en-tiempos-de-covid-19-clinica-evolucion-y-hallazgos-de-laboratorio

\section{Características}

Entre las características principales de las lesiones dermatológicas en pacientes pediátricos con infección por SARS-CoV-2, Ordoñez \& Domínguez (2020) destacan las siguientes:

- Estas lesiones son dolorosas, rojas o a veces negruzcas.

- Pueden ser redondeadas, de unos pocos milímetros, múltiples o extenderse a todo un dedo, generalmente con una clara demarcación a nivel metatarso falángico.

- Usualmente afectan a tres dedos, a menudo separados por otros no afectados.

- Inicialmente tienen un color rojo violáceo o azulado; pueden volverse bullosas o tener costras negruzcas.

- Tienen un curso bastante duradero, in- 
NO, M. I.

cluso durante dos semanas.

- Se asocian varios casos dentro de los convivientes o grupo familiar.

\section{Tratamiento}

"El tratamiento es sintomático, pudiendo manejarse con corticoides tópicos, antihistamínicos en el caso de prurito moderado, y antibióticos tópicos para evitar sobreinfecciones en las lesiones acroisquémicas" Maqueda et al. (2020).

Por su parte Romo et al. (2020) de igual manera refiere que el tratamiento de las manifestaciones cutáneas es fundamentalmente sintomático y hace alusión a las siguientes indicaciones, según el tipo de lesión:

- En el caso del exantema, erupción urticariforme o erupción vesiculosa, se pueden pautar antihistamínicos, emolientes, antisépticos y corticoides tópicos en caso de sintomatología importante.

- En las lesiones acroisquémicas, se aconseja observación clínica de las lesiones, evitar el frío y si son muy sintomáticas se ha recomendado nitroglicerina tópica $0.2 \%$, diltiazem $2 \%$ en gel, trinitrato de glicerol $4 \mathrm{mg} / \mathrm{g}$, corticoides tópicos (controvertido) o emolientes con lanolina y glicerina.

Por lo general el pronóstico es bueno, auto limitándose en el tiempo las lesiones, sin aumentar las complicaciones ni mortalidad del COVID-19, por lo que no consideraríamos las complicaciones dermatológicas como factores de mal pronóstico de la enfermedad.

\section{Conclusión}

El virus SARS-CoV-2 causante de la pandemia por COVID-19 que ha afectado al mundo en los últimos dos años, se caracteriza por ser una infección de tracto respiratorio, cuyos síntomas son muy parecidos a los del resfriado común y que suelen ser más leves en pacientes pediátricos que en adultos.
Además de los síntomas que afectan el tracto respiratorio, se han desarrollado una serie de lesiones dermatológicas en pacientes afectados por esta patología, entre las que destacan, lesiones pseudosabañón, erupciones vesiculares, lesiones tipo urticaria, erupciones maculopapulares y livedo o necrosis.

El tratamiento para estas lesiones cutáneas es fundamentalmente sintomático, pudiendo manejarse con corticoides tópicos, antihistamínicos en el caso de prurito moderado, y antibióticos tópicos.

Aun y cuando este tipo de lesiones no representan una amenaza grave para los pacientes, se recomienda conocer la sintomatología de las mismas para así aplicar las medidas preventivas y de tratamiento que eviten posibles complicaciones.

\section{Bibliografía}

Andina, D., Noguera, L., \& Bascuas, M. (2020). Intramed. Recuperado el 22 de 07 de 2021, de Intramed: https://www.intramed.net/contenidover.asp?contenidoid $=96452 \&$ pagina $=1$

Benavides , I., Lopez, E., \& Lopez, P. (2020). Diferencias entre niños y adultos por el nuevo coronavirus 2019, SARS-CoV-2/COVID-19. Revista Latinoamericana de Infectología Pediátrica, 33(4), 165-173. doi:doi: 10.35366/96845

Granados, L., Broche, L., Perez, L., \& Lopez, V. (2020). Manifestaciones cutáneas en pacientes pediátricos infectados por el coronavirus SARSCoV-2. Revista Cubana de Pediatría., 92. Recuperado el 23 de 07 de 2021, de http://www.revpediatria.sld.cu/index.php/ped/article/view/1171/556

Maqueda, G., Sierra, L., Sierra, E., \& Martinez, J. (21 de 09 de 2020). Manifestaciones dermatológicas de la infección por COVID-19 en Pediatría. Revista Clínica de Medicina de Familia, 13(2). Recuperado el 23 de 07 de 2021, de https:// scielo.isciii.es/scielo.php?script=sci_arttext\&pi$d=S 1699-695 X 2020000200013$

Mayo Clinic. (24 de 03 de 2021). Mayo Clinic. Recuperado el 19 de 07 de 2021, de Mayo Clinic: https://www.mayoclinic.org/es-es/diseases-conditions/mis-c-in-kids-covid-19/symptoms-causes/ syc-20502550 
Montaño, V., \& Miranda, M. (01 de 2021). Actualización del manejo clínico de COVID-19 en pediatría: a un año de pandemia. Revista Mexicana de Pediatria, 88(1), 31-45. doi:doi: 10.35366/99417

Oliva, J. (22 de 03 de 2021). COVID-19 en la niñez y adolescencia. Revista Cientifica del Instituto Nacional de la Salud, 4(1), 49-61. doi:DOI 10.5377/ alerta.v4i1.9780

OPS. (18 de 05 de 2021). Organizacion Panamericana de la Salud (PAHO). Recuperado el 20 de 07 de 2021, de Organizacion Panamericana de la Salud (PAHO): https://www.paho.org/es/file/88667/ download?token=bFaWY4XT

Ordoñez, M., \& Dominguez, B. (18 de 09 de 2020). Acroisquemia aguda en tiempos de COVID-19. Clínica, evolución y hallazgos de laboratorio. Pediatria Atencion Primaria, 2(87), 295-303. Recuperado el 21 de 07 de 2021, de https://pap. es/articulo/13107/acroisquemia-aguda-en-tiempos-de-covid-19-clinica-evolucion-y-hallazgos-de-laboratorio

Reyes, A. (2021). Manifestaciones clínicas no respiratorias, secundarias a infección por SARS-CoV-2 en niños. Acta Pediatrica Mexico, 42(1), 48-52. Recuperado el 22 de 07 de 2021, de https://ojs.actapediatrica.org.mx/index.php/APM/article/down$\mathrm{load} / 2215 / 1234$
Romo, K., Saucedo, E., Hinojosa, S., Mercado, J., Uc, J., Ochoa, E., ... . Padilla, T. (2020). Manifestaciones clínicas de la COVID-19. Revista Latinoamericana de Infectología Pediátrica, 33(1), 10-32. doi:doi: 10.35366/96668

Ruiz, A., \& Jimenez, M. (30 de 03 de 2020). SARSCoV-2 y pandemia de síndrome respiratorio agudo (COVID-19). Ars Pharmaceutica, 61(2), 63-79. doi:https://dx.doi.org/10.30827/ars.v61i2.15177

Solano, A., Solano, A., \& Gamboa, C. (07 de 2020). SARS-CoV-2: la nueva pandemia. Revista Médica Sinergia, 5(7). doi:https://doi.org/10.31434/rms. v5i7.538

Vargas, A., Schreiber, V., Ochoa , E., \& Lopez, A. (07 de 2020). SARS-CoV-2: una revisión bibliográfica de los temas más relevantes y evolución del conocimiento médico sobre la enfermedad. Neumología y Cirugía de Tórax, 79(3), 185-196. doi:doi: $10.35366 / 96655$

\section{CITAR ESTE ARTICULO:}

Moreira Quiroz, V. del R., Romero Tejada, C. A., Zambrano Zambrano, M. D., \& Zambrano Zambrano, M. I. (2021). Lesiones dermatológicas en pediatría por infección SARS-CoV-2. RECIAMUC, 5(3), 36-43. https://doi.org/10.26820/

reciamuc/5.(3).agosto.2021.36-43

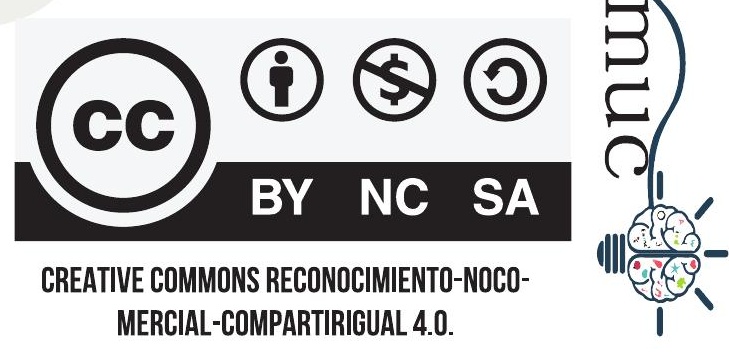

